\title{
Generation of a few femtosecond keV x-ray pulse via interaction of a tightly focused laser copropagating with a relativistic electron bunch
}

\author{
Sang-Young Chung and Hae June Lee \\ Department of Electrical Engineering, Pusan National University, San 30, Jangjeon-dong, Geumjeong-gu, Busan 609-735, Korea \\ Kitae Lee \\ Quantum Optics Division, Korea Atomic Energy Research Institute, 1045 Daedeok-daero, Yuseong, Daejeon 305-353, Korea

$$
\text { Dong Eon Kim* }
$$ \\ Department of Physics, POSTECH, San 31 Hyoja-dong, Nam Ku, Pohang, Kyungbuk 790-784, Korea
} (Received 22 April 2009; revised manuscript received 29 September 2010; published 17 June 2011)

\begin{abstract}
It is demonstrated in a numerical simulation that an intense fs keV x-ray pulse can be generated by the interaction of a tightly focused femtosecond laser copropagating with an electron bunch. In general, the interaction of a loosely focused (focal spot $\sim 100 \mu \mathrm{m}$ in diameter) laser with a copropagating electron is rather weak so that the radiation is not only weak but also produced in the vicinity of laser wavelength. However, in the case of tight focus (focal spot on the order of wavelength), the radiation characteristics turn out to be drastically different so that a $\mathrm{keV}$ x-ray pulse can be produced at high flux. This is due to the nonparaxial fields induced in a tight-focus regime. The radiation characteristics are discussed for different beam waists and electron beam energies. This simulation suggests that the interaction of a tightly focused laser with a copropagating electron bunch can be a unique source for an x-ray pulse of the photon energy from 10 to $100 \mathrm{keV}$ that lasts a few femtoseconds.
\end{abstract}

DOI: 10.1103/PhysRevSTAB.14.060705

PACS numbers: 42.65.Re, 42.68.Mj, 41.50.+h

\section{INTRODUCTION}

Ultrafast high-intensity $\mathrm{x}$ rays are useful for investigation of ultrafast phenomena in atomic and molecular physics, plasma physics, material science, chemistry, and life science $[1,2]$. They can be used to observe atomic structure and dynamics [3], to investigate ultrafast phase transition in solids [4], and to produce time-resolved biomolecular images [5]. Ultrafast high-intensity x rays can be generated from the interaction of a high-intensity femtosecond (fs) laser by Compton backscattering [6], relativistic nonlinear Thomson scattering [7-9], and laser-produced betatron radiation [10,11]. In synchrotron facilities, electron bunch slicing has been adopted for experiments [12-14]. X-ray free-electron lasers (XFELs) [15-17] are becoming available. Schemes for subfemtosecond or attosecond (as, $10^{-18}$ second) $\mathrm{x}$-ray sources based on XFEL have been proposed [18-21]. No currently available ultrafast x-ray source can produce both pulses $<80 \mathrm{fs}$ and energies $\geq 10 \mathrm{keV}$ (Fig. 1). However, many processes in nature occur on time scales of a few femtoseconds that can only be investigated using photons with kiloelectronvolt energies $[22,23]$. Therefore, demands are increasing for pulses

\footnotetext{
*kimd@ postech.ac.kr

Published by the American Physical Society under the terms of the Creative Commons Attribution 3.0 License. Further distribution of this work must maintain attribution to the author(s) and the published article's title, journal citation, and DOI.
}

$<10 \mathrm{fs}$ in this spectral region. This paper simulates generation of such a short pulse from the interaction of a femtosecond laser with an electron beam.

In the case of the interaction of an electron beam with a laser, there are three major interaction geometries: counterpropagation (Compton backscattering), $90^{\circ}$ scattering, and copropagation $\left(0^{\circ}\right.$ scattering) geometry. Figure 2(a) shows the copropagation interaction geometry. Estimating the

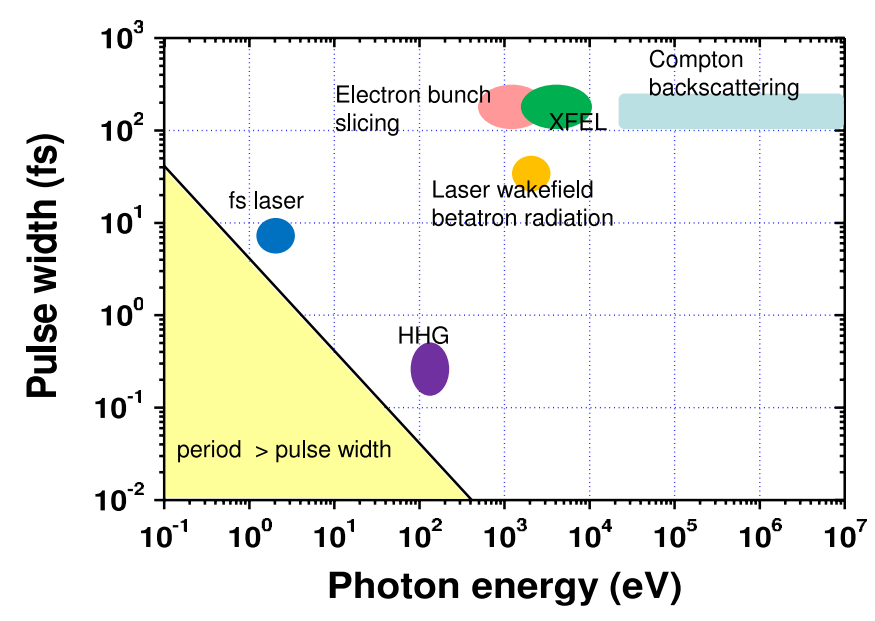

FIG. 1. Pulse duration and photon energy of currently available ultrafast light sources: Compton backscattering [6], laser wakefield betatron radiation [10,11], electron bunch slicing [12-14], and XFEL [15-17]. No existing source produces ultrafast ( $<80 \mathrm{fs}$ ) with energies $\geq 10 \mathrm{keV}$. 
pulse widths $(\Delta t)$ of the radiations in these interaction geometries requires values of the length $\left(L_{e}\right)$ and diameter $\left(L_{T}\right)$ of the electron bunch, the pulse length $\left(L_{\text {laser }}\right)$ of the driving laser, and the interaction length (confocal parameter, $L_{\text {conf }}$ ). For current technology, the typical pulse width of a relativistic electron bunch is $\sim 100 \mathrm{fs}$ and that of a femtosecond laser is $\sim 5 \mathrm{fs}$; these dimensions correspond to $L_{e} \sim 30 \mu \mathrm{m}$ and $L_{\text {laser }} \sim 1.5 \mu \mathrm{m}$. When the beam waist at focus is $5 \mu \mathrm{m}$ and laser wavelength is $800 \mathrm{~nm}$, the confocal parameter is $L_{\text {conf }} \sim 100 \mu \mathrm{m}$; i.e., the condition $L_{\text {conf }} \gg L_{e} \gg L_{\text {laser }}$ is satisfied. Here we confine our interest to this case. $L_{T}$ is typically $30 \mu \mathrm{m}$. In this situation, $\Delta t \sim 2 L_{e} / c=200 \mathrm{fs}$ for the counterpropagation, $\Delta t \sim$ $L_{T} / c=100 \mathrm{fs}$ for the $90^{\circ}$ scattering, and $\Delta t \sim L_{\text {laser }} / c=$ $5 \mathrm{fs}$ for the copropagation geometry, where $c$ is the speed of light in a vacuum.

The estimated pulse widths of the counterpropagating and the copropagating interactions can be explained as follows. In the counterpropagating interaction the entire electron bunch interacts with the laser pulse while it passes through the focused region [Fig. 2(b)]. The entire bunch radiates X-rays and the pulse length is almost the same as the bunch length. In contrast, in the copropagating interaction only a small part of the electron bunch can interact with the strong laser pulse in the focused region [Fig. 2(c)] because the relative velocity between the bunch and the laser is very small. For example, when the energy of the electron is $200 \mathrm{MeV}$, the relative velocity is just $1 \mathrm{~km} / \mathrm{s}$ and the displacement increase while it passes the Rayleigh range is $<0.7 \mathrm{~nm}$. The length of the part of the bunch that interacts with the laser and the length of the radiation pulse are almost the same as the length of the laser pulse. The remainder of the bunch cannot intersect the strong part of the laser pulse in the focused region; even though this part of the bunch can interact with the laser at a distance from the focused region, the laser field is much weaker there than in the focused region. When the center of the electron bunch meets the peak of the laser pulse at the center of the focus, an electron that is $3 \mu \mathrm{m}$ ( $10 \mathrm{fs}$ in time scale) from the center of the bunch meets the peak at $0.9 \mathrm{~m}$ from the center of the focus. The interaction is negligible because it is too far from the focus.

The copropagation geometry can generate the shortest $\mathrm{x}$-ray pulse among the interaction geometries. However, the copropagating interaction of a loosely focused (focal spot $\sim 100 \mu \mathrm{m}$ in diameter) laser produces weak radiation that has the same wavelength as that of the laser due to the Doppler effect.

The radiation from a tightly focused laser with a copropagating electron has radiation characteristics that are totally different from those of a loosely focused laser interacting with an electron beam [24,25]; when a laser is tightly focused, the paraxial approximation (the electric and magnetic field transverse to the propagation direction) is not valid $[26,27]$ because the electric and magnetic field

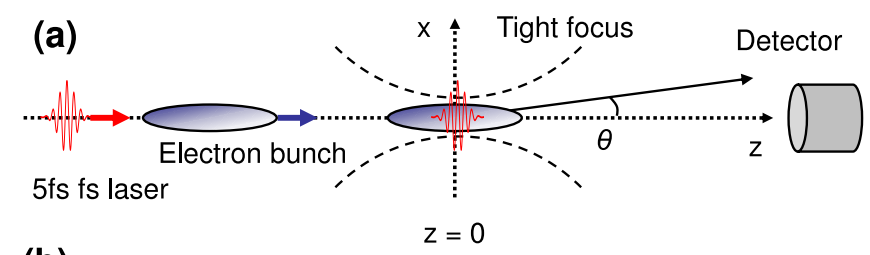

(b)
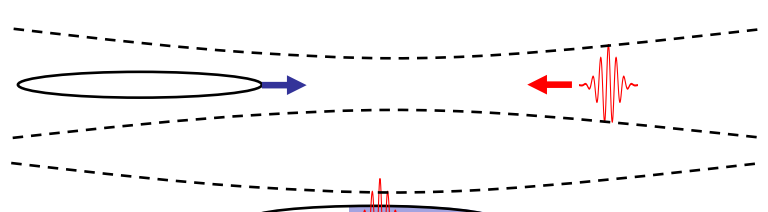

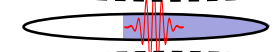

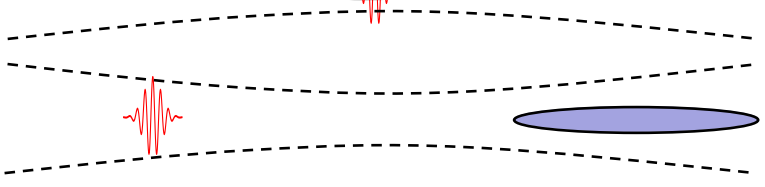

(c)

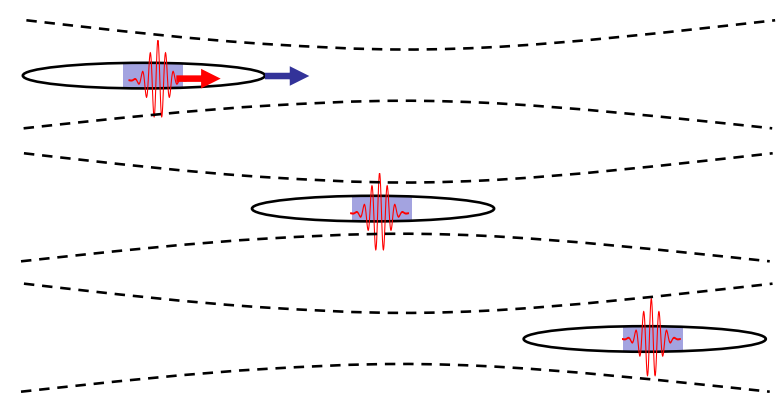

FIG. 2. (a) Schematic diagram of the interaction of a femtosecond laser copropagating with an electron bunch. Both the laser and the electron bunch propagate along the $+z$ direction. $z=0$ is defined to be at the best focus of the laser. The detector is located far away in the $+z$ direction on the axis $(\theta=0)$. (b), (c) The changes of the position of the electron bunch, the laser pulse, and the focused region (b) in the counterpropagating interaction and (c) in the copropagating interaction. The filled parts of the electron bunch indicate the part that is or has interacted with the strong laser field.

have nonparaxial components, some of which are parallel to the propagation direction. The temporal and spatial shapes of the electric field are not the same as those of the magnetic field.

In the copropagating interaction of a relativistic electron with a paraxial laser field, the magnitude of the force from the magnetic field is similar to that of electric field but in the opposite direction. The forces cancel each other, so the acceleration is very small and the radiation is weak. However, in a tightly focused laser field, the shape of the magnetic field is different from that of the electric field and significant force is left when the forces are added. The radiation is much stronger than that of loosely focused laser field or paraxial field.

When the Doppler effect is considered, an electron interacting with a copropagating laser field radiates photons whose energy is the same as the photon energy of the laser. However, the photon energy of the radiation cannot 
be calculated by this manner with the tightly focused laser. When an electron of $200 \mathrm{MeV}$ energy copropagates with a laser of $800 \mathrm{~nm}$ wavelength, the electron must travel $0.24 \mathrm{~m}$ to interact with one cycle of the laser field wave. When the beam waist of the laser is $5 \mu \mathrm{m}$, the Rayleigh length is $\sim 100 \mu \mathrm{m}$. The electron interacts with about $1 / 1000$ of the laser cycle while it passes through the Rayleigh range. When the electron interacts with $1 / 25$ of the wave cycle, the laser intensity becomes $10^{-4}$ times the intensity at the center of focus. Because the electron cannot interact with one entire laser wave cycle, the electron cannot recognize the photon energy of the laser and it does not radiate photons with the laser photon energy. The copropagating interaction with the tightly focused laser is similar to wiggler interaction. While the electron copropagates with the tightly focused laser, it interacts with the strong field only in the Rayleigh range and the temporal change of the field is negligible. It can be considered as a very short electric field wiggler. Because the interaction length corresponding to the wiggler length is just several hundred micrometers, $\mathrm{keV} \mathrm{x}$-ray radiation can be achieved from the copropagating interaction. The nonparaxial laser field shortens the length of the "wiggler" and increases the photon energy.

In this paper, we simulate the effect of this nonparaxial field of the laser field on the radiation from an electron bunch interacting with a copropagating tightly focused femtosecond laser. Simulation results suggest that these nonparaxial elements of the laser field make significant contributions so that $\mathrm{keV} \mathrm{x}$-ray photons are produced at a high photon flux. Nonparaxial fields and basic formulations are briefly described in Sec. II. Simulation results are presented, discussed, and compared with the currently available femtosecond x-ray sources in Sec. III. Findings suggest that the interaction of a tightly focused femtosecond laser copropagating with a currently available electron bunch can produce strong $\mathrm{keV}$ x-ray pulses of a few femtosecond duration.

\section{THEORY}

Consider the case (Fig. 2) in which an electron bunch and a pulsed laser copropagate along the $+z$ direction and meet each other in a tightly focused region. The laser is polarized in the $x$ direction. The electron bunch under consideration is relativistic: $\beta \simeq 1$, where $\beta=|\vec{\beta}|=$ $|\vec{v} / c|$ and $\vec{v}$ is the velocity of an electron. In this section we will derive the properties of the radiation from the interaction of a tightly focused laser pulse with a copropagating electron bunch.

In the tightly focused laser field, the paraxial approximation is not valid because it does not agree with the Maxwell equation. Figure 3 shows the paraxial approximation of a linearly polarized tightly focused laser field. When a thin and narrow box as shown Fig. 3 is considered, the electric field is stronger at the bottom of the region than

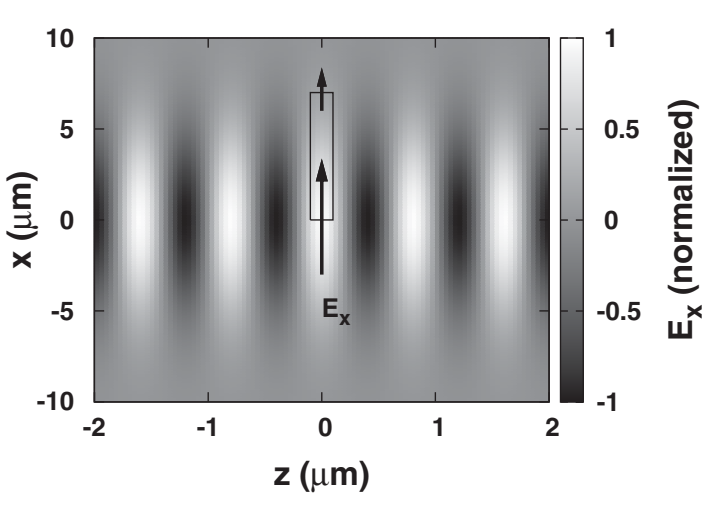

FIG. 3. Paraxial approximation of the tightly focused laser field. The field is polarized in the $x$ direction, the beam waist is $5 \mu \mathrm{m}$, and the wavelength is $800 \mathrm{~nm}$. The rectangle in the graph indicates a thin and narrow box. The electric field (arrows) on the bottom of the box is stronger than the electric field on the top. Because the box does not include any charge, the paraxial approximation disagrees with Gauss's law.

at the top, and all electric fields are parallel to the sides. The total electric flux through the box surface is not zero but no charge occurs within it. This shows that the paraxial approximation cannot satisfy Gauss's law. To satisfy Gauss's law and the Maxwell equation, other electric fields that are orthogonal to the paraxial electric field are required. The tightly focused laser field that satisfy the Maxwell equation can be described using an infinite power series of diffraction angle $\varepsilon=w_{0} / z_{r}$, where $w_{0}$ is beam waist and $z_{r}=\pi w_{0}^{2} / \lambda$ is Rayleigh range, where $\lambda$ is the wavelength of the laser. In this study, the tightly focused laser field derived in $[28,29]$ was used. Fields up to 7 th order in $\varepsilon$ were considered in the simulation. The validity of this limit of the order will be confirmed in this and the next section. The fields $E_{x}, B_{y}$, and $E_{z}$ are written here up to the 2nd order of $\varepsilon$, for simplicity:

$$
\begin{gathered}
E_{x}=E\left[S_{1}+\varepsilon^{2}\left(\xi^{2} S_{3}-\frac{\rho^{4} S_{4}}{4}\right)+\cdots\right] \\
B_{y}=E\left[S_{1}+\varepsilon^{2}\left(\frac{\rho^{2} S_{3}}{2}-\frac{\rho^{4} S_{4}}{4}\right)+\cdots\right] \\
E_{z}=E \xi\left[\varepsilon\left(C_{2}\right)+\cdots\right],
\end{gathered}
$$

where $\quad E=E_{1} \exp \left(-r^{2} / w^{2}\right), \quad r^{2}=x^{2}+y^{2}, \quad w=$ $w_{0} \sqrt{1+\left(z / z_{r}\right)^{2}}, \quad \xi=x / w_{0}, \quad$ and $\quad \rho^{2}=r^{2} / w_{0}^{2} . \quad E_{1}=$ $E_{0} \exp \left[-2 \ln 2(t-z / c)^{2} / \Delta t_{L}^{2}\right]$, where $E_{0}$ is the peak electric field and $\Delta t_{L}$ the laser pulse duration at full-width half-maximum (FWHM). $C_{n}$ and $S_{n}$ are defined as

$$
\begin{gathered}
C_{n}=\left(\frac{w_{0}}{w}\right)^{n} \cos \left(\psi+n \psi_{G}\right) ; \quad n=1,2,3 \ldots \\
S_{n}=\left(\frac{w_{0}}{w}\right)^{n} \sin \left(\psi+n \psi_{G}\right),
\end{gathered}
$$


where $\quad \psi=\psi_{0}+\omega t-k z-k r^{2} /(2 R) \quad$ and $\quad R=$ $z\left[1+\left(z_{r} / z\right)^{2}\right] . \psi_{0}$ is a constant initial phase, $k=2 \pi / \lambda$, and $\omega=2 \pi c / \lambda . \psi_{G}$ is Gouy phase expressed as

$$
\psi_{G}=\tan ^{-1} \frac{z}{z_{r}} .
$$

Because the field of the lowest order, $\varepsilon^{0}$, is identical to the paraxial field, the other fields will be called high-order fields (HOFs) in this study.

The total radiated power from an accelerated electron is described as

$$
P\left(t^{\prime}\right)=\frac{2}{3} \frac{e^{2}}{c} \gamma^{6}\left[(\dot{\vec{\beta}})^{2}-(\vec{\beta} \times \dot{\vec{\beta}})^{2}\right]
$$

where $t^{\prime}$ is the retarded time and $\dot{\vec{\beta}}$ the acceleration of the electron divided by $c$ [30]. $e$ is the electron charge and $\gamma$ is Lorentz's factor, $\left(1-\beta^{2}\right)^{-1 / 2}$. The radiated power in the electron's own time (retarded time) $P\left(t^{\prime}\right)$ and in the detector's own time $P(t)$ are related to each other as follows:

$$
\begin{aligned}
P(t) & =\int_{\theta<\pi} \frac{d P(t)}{d \Omega} d \Omega \simeq \int_{\theta<1 / \gamma} \frac{d P(t)}{d \Omega} d \Omega \\
& =\int_{\theta<1 / \gamma} \frac{d P\left(t^{\prime}\right)}{d \Omega} \frac{d t^{\prime}}{d t} d \Omega \\
& =\int_{\theta<1 / \gamma} \frac{d P\left(t^{\prime}\right)}{d \Omega} \frac{1}{1-\hat{n} \cdot \vec{\beta}} d \Omega \\
& \simeq \int_{\theta<1 / \gamma} \frac{d P\left(t^{\prime}\right)}{d \Omega} \frac{1}{1-\beta} d \Omega \\
& =\frac{1}{1-\beta} \int_{\theta<1 / \gamma} \frac{d P\left(t^{\prime}\right)}{d \Omega} d \Omega \simeq \frac{P\left(t^{\prime}\right)}{1-\beta},
\end{aligned}
$$

where $\theta$ is the angle from the $z$ axis [Fig. 2(a)]. For the relativistic electron bunch used in this study a great part of the radiation is detected at $\hat{n} \simeq \vec{\beta} / \beta$ (or $\theta<1 / \gamma$ ), so $(1-\hat{n} \cdot \vec{\beta})$ can be considered as $\sim(1-\beta)$. Because $\beta \simeq 1,(1-\beta) \simeq 1 /\left(2 \gamma^{2}\right)$, so $P(t) \simeq 2 \gamma^{2} P\left(t^{\prime}\right)$.

When $\dot{\vec{\beta}}$ is parallel to $\vec{\beta}$, the radiated power integrated over all the angles is given by

$$
P_{\|}=\frac{4}{3} \frac{e^{2}}{c} \gamma^{8} \dot{\beta}_{\|}^{2} .
$$

On the other hand, when $\dot{\vec{\beta}}$ is perpendicular to $\vec{\beta}$ the radiation power is expressed as

$$
P_{\perp}=\frac{4}{3} \frac{e^{2}}{c} \gamma^{6} \dot{\beta}_{\perp}^{2}
$$

$\dot{\vec{\beta}}$ can be obtained from a relativistic equation of motion $d(\gamma \vec{\beta}) / d t=(e / m c) \vec{F}$ where $\vec{F}=\vec{E}+\vec{\beta} \times \vec{B}$; here we call this the effective field. The left side of the equation of motion can be expanded as $\gamma \dot{\vec{\beta}}+\gamma^{3} \beta \vec{\beta} \dot{\beta}$. The magnitude of the second term $\gamma^{3} \beta \vec{\beta} \dot{\beta}$ is much larger than that of the first term and makes the major contribution to $\vec{F}_{\|}$ parallel to $\vec{\beta}$. The main contribution to $\vec{F}_{\perp}$ perpendicular to $\vec{\beta}$ comes from the $\gamma \dot{\vec{\beta}}$ term. From this relation, $\dot{\beta}$ can be expressed with $\vec{F}$ and $\gamma$; thus Eqs. (9) and (10) can be rewritten as

$$
P_{\|}(t) \simeq \frac{4}{3} \frac{e^{4}}{m c^{3}} \gamma^{8}\left|\frac{\vec{F}_{\|}}{\beta^{2} \gamma^{3}}\right|^{2} \simeq \frac{4}{3} \frac{e^{4}}{m c^{3}} \gamma^{2}\left|\vec{F}_{\|}\right|^{2}
$$

and

$$
P_{\perp}(t) \simeq \frac{4}{3} \frac{e^{4}}{m c^{3}} \gamma^{6}\left|\frac{\vec{F}_{\perp}}{\gamma}\right|^{2}=\frac{4}{3} \frac{e^{4}}{m c^{3}} \gamma^{4}\left|\vec{F}_{\perp}\right|^{2},
$$

respectively.

When a single electron copropagates with a tightly focused laser pulse, a short radiation pulse is generated because the electron is strongly accelerated and radiates photons only in the focused region. When an electron passes with a speed $c \beta$ through an interaction region of length $\Delta z$, which is similar and proportional to $z_{r}$ and radiates in the direction $\hat{z}$, the difference between the arrival times of the beginning and the end of the radiation at the detector located far away on the $+z$ axis is

$$
\Delta t_{S}=\frac{\Delta z(1-\beta)}{c \beta} \simeq \frac{\Delta z}{2 c \gamma^{2}} \simeq \frac{z_{r}}{c \gamma^{2}}=\frac{\pi}{c \lambda} \frac{w_{0}^{2}}{\gamma^{2}}
$$

because $\beta \simeq 1$. The time difference $\Delta t_{S}$ is the duration of the radiated pulse from a single electron, approximately. $\left(\Delta t_{S}\right.$ will be discussed more accurately later in this section.) For a laser with $\lambda=800 \mathrm{~nm}$ and a beam waist of $5 \mu \mathrm{m}, \Delta t_{S} \sim 2.1$ as for an electron energy of $200 \mathrm{MeV}$. This time range is much smaller than the pulse width of the whole bunch ( $\Delta t \simeq \Delta t_{L}=5 \mathrm{fs}$ ), so it does not affect the final pulse width. Using this $\Delta t_{S}$, the total radiation energy of single electron $I_{S}$ can be estimated from Eqs. (11) and (12) as follows:

$$
\begin{gathered}
I_{S \|} \simeq \frac{4}{3} \frac{\pi e^{4}}{m c^{4} \lambda} w_{0}^{2}\left|\vec{F}_{\|}\right|^{2} \\
I_{S \perp} \simeq \frac{4}{3} \frac{\pi e^{4}}{m c^{4} \lambda} w_{0}^{2} \gamma^{2}\left|\vec{F}_{\perp}\right|^{2} .
\end{gathered}
$$

For a uniform distribution of electrons in an electron bunch, the total radiation energy from an electron bunch $I$ is

$$
\begin{gathered}
I_{\|} \propto w_{0}^{4}\left|\vec{F}_{\|}\right|^{2} \\
I_{\perp} \propto w_{0}^{4} \gamma^{2}\left|\vec{F}_{\perp}\right|^{2} .
\end{gathered}
$$

When only the 0th order field is included, the effective field is perpendicular to the laser propagation, and from Eqs. (1) and (2) 


$$
\vec{F}_{(0)}=E S_{1}(1-\beta) \hat{x} \simeq \frac{E S_{1}}{2 \gamma^{2}} \hat{x} .
$$

Substituting Eq. (18) into Eq. (17) yields

$$
I_{(0)} \propto\left(w_{0}^{4} \gamma^{2}\right)\left(\varepsilon^{0} \gamma^{-4}\right) \propto w_{0}^{4} \gamma^{-2} .
$$

For the 1st order field from Eq. (3), the effective field can be written as

$$
\vec{F}_{(1)}=E \xi \varepsilon C_{2} \hat{z} .
$$

The total radiated energy by the 1 st order field is

$$
I_{(1)} \propto w_{0}^{4}\left(\varepsilon^{2} \gamma^{0}\right) \propto w_{0}^{2} \gamma^{0}
$$

using Eqs. (20) and (16). With respect to the 2nd order field from Eqs. (1) and (2), the effective field is given by

$$
\begin{aligned}
\vec{F}_{(2)} & =E \varepsilon^{2}\left[-(1-\beta) \frac{\rho^{4} S_{4}}{4}+\left(\xi^{2}-\frac{\rho^{2}}{2} \beta\right) S_{3}\right] \hat{x} \\
& \simeq E \varepsilon^{2} S_{3}\left(\xi^{2}-\frac{\rho^{2}}{2}\right) \hat{x} .
\end{aligned}
$$

For relativistic electrons $1-\beta \simeq 0$, so the total radiated energy by the 2 nd order field is

$$
I_{(2)} \propto\left(w_{0}^{4} \gamma^{2}\right)\left(\varepsilon^{4} \gamma^{0}\right) \propto w_{0}^{0} \gamma^{2}
$$

using Eqs. (22) and (17).

Because $\Delta t_{S}\left(\propto w_{0}^{2} \gamma^{-2}\right)$ and the participating electron number ( $\propto w_{0}^{2}$ ) are common for Eqs. (19), (21), and (23), the ratio between $I_{(0)}, I_{(1)}$, and $I_{(2)}$ is approximately $1: \varepsilon^{2} \gamma^{2}: \varepsilon^{4} \gamma^{4}$. Because $1 / \varepsilon^{2}$ only $\sim 4 \times 10^{2} \ll \gamma^{2}$ ( $\gamma^{2} \geq 10^{4}$ in the current study) for $\lambda=800 \mathrm{~nm}$ and a beam waist of $5 \mu \mathrm{m}, I_{(0)} \ll I_{(1)} \ll I_{(2)}$. The effect of $\xi$ and $\rho$ are averaged out in the case of an electron bunch, and do not have significant effect on the difference among the total radiated energies $I_{(0)}, I_{(1)}$ and $I_{(2)}$.

The result of $I_{(0)} \ll I_{(1)} \ll I_{(2)}$ can be explained as follows: the effective fields of the 0th order fields are very small because the 0th order electric field and magnetic field cancel each other in the copropagation geometry for a relativistic electron beam. Even though the 0th fields themselves are stronger than other higher-order fields, the effective field $\vec{F}_{(0)}$ of the 0 th order field is much smaller than $\vec{F}_{(1)}$ and $\vec{F}_{(2)}$. Hence, $I_{(0)} \ll I_{(1)}, I_{(2)}$. On the other hand, $I_{(1)}<I_{(2)}$ because a transverse field induces radiation more effectively than does a longitudinal field. The effective fields, whose order is higher than the 2 nd order, do not make significant contributions to radiation because their strengths are smaller than that of the 2nd order field.

A single electron radiates a very short pulse [Eq. (13)]. Such a short pulse has a large spectral bandwidth. The maximum photon energy is roughly proportional to the inverse of the pulse width when the pulse is transform limited. From Eq. (13), the following dependency is derived:

$$
K_{\max } \propto \frac{1}{\Delta t_{S}} \simeq \frac{2 c \gamma^{2}}{\Delta t_{S}} \simeq \frac{c \gamma^{2}}{z_{r}}=c \gamma^{2} \frac{\lambda}{\pi w_{0}^{2}} \propto \frac{\gamma^{2}}{w_{0}^{2}}
$$

when $K_{\max }$ is the maximum photon energy. Because a short radiation pulse can be generated by a short Rayleigh length, tight focusing alone can increase the photon energy without the HOFs. In addition, the HOFs can increase the photon energy in two ways. First, they decrease the interaction region (i.e. effective Rayleigh length). The strength of field is proportional to $\left(w_{0} / w\right)^{n}=\left[1 / \sqrt{1+\left(z / z_{r}\right)^{2}}\right]^{n}$ [Eqs. (4) and (5)]; this indicates that FWHM length in the intensity $\Delta z$ is $2 z_{r} \sqrt{2^{1 / n}-1}$. Hence, the magnitude of the 2 nd order field [ $S_{3}, n=3$, Eq. (22)] is 0.51 times that of the paraxial field $\left[S_{1}, n=1\right.$, Eq. (18)]. Notice that the number $n$ is not the order of the HOFs but a parameter related to $S_{n}$ and $C_{n}$. Reducing the interaction region reduces pulse length and increases photon energy. Second, the HOFs increase the speed of oscillations in the radiation. The phases of $S_{n}$ and $C_{n}$ consist of a constant phase $\phi_{0}$, a plane wave phase $\omega t-k z$, a radial phase $-k r^{2} /(2 R)$, and a Gouy phase $n \psi_{G}$. Because the velocity of the copropagating electron is almost the same as the speed of light, the plane wave phase $\omega t-k z$ is almost constant while the electron passes through the Rayleigh range. When a relativistic electron copropagates with the laser, the plane wave phase is

$$
\omega t-k z \simeq k z(1-\beta) \simeq \frac{k z}{2 \gamma^{2}}=\frac{\pi z}{\lambda \gamma^{2}} .
$$

When $w_{0}=5 \mu \mathrm{m}$ and $\gamma=100$, the change of the plane wave phase is $\leq 0.08$ while the Gouy phase changes by $n \pi / 2$. Because the plane wave phase can be ignored, the electron is affected only by the phase of $-k r^{2} /(2 R)+$ $n \psi_{G}$ and both of them depend only on the position of the electron. $-k r^{2} /(2 R)$ is independent of the order of the field but $n \psi_{G}$ changes increasingly rapidly as order increases: by $\pi$ in the 0th order field, $2 \pi$ in the 1 st order, $3 \pi$ in the 2nd order field, and so on. The change of the phase means a change in the direction of the field affecting the electron. The electron oscillates motion while it traverses the Rayleigh region; the oscillations become more rapid as the order of field increases (Fig. 4). It is difficult to recognize because the envelope becomes shorter simultaneously. When the envelope and field [Figs. 4(a) and 4(b)] are compared more closely, the width of the oscillation is shorter than that of the envelope by the phase $n \psi_{G}$ in 2nd order fields but the two widths are the same in a paraxial field. In addition to the shorter envelop, the more rapid oscillations also cause higher photon energy. This phenomenon can also be understood in another. Because the field almost depends on the position only, the change of the field can be considered a very short wiggler with a length $\sim 50 \mu \mathrm{m}$ in the case of the $2 \mathrm{nd}$ order field. The length of one period of the wiggler decreases in 

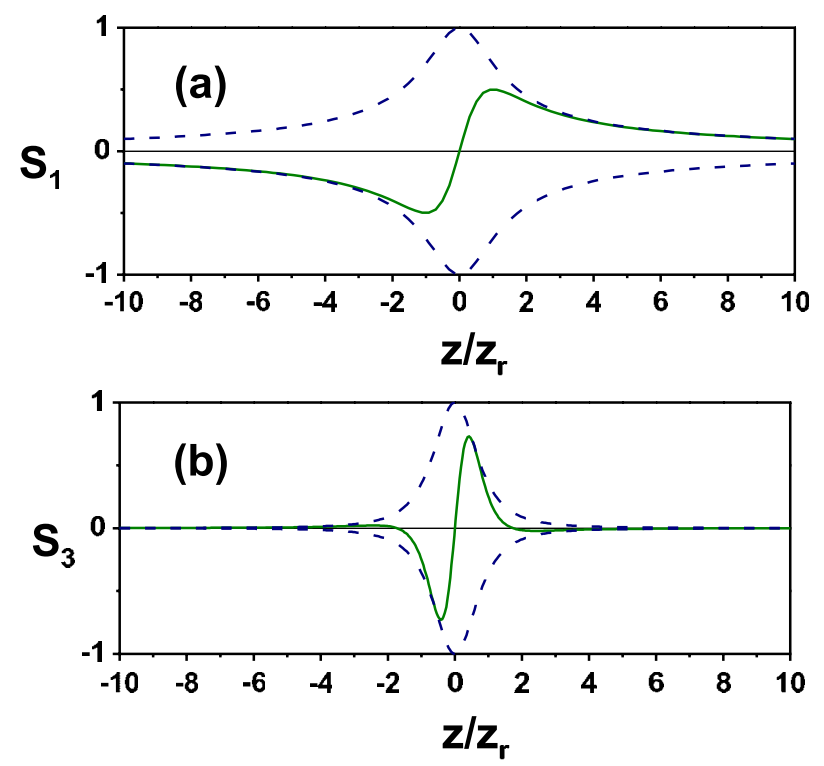

FIG. 4. (a) $S_{1}$ and (b) $S_{3}$, described in Eq. (5) when $r=0$ and $\omega t-k z=0 . S_{1}$ is the paraxial field and $S_{3}$ field and 2nd order field, respectively. The dashed lines indicate $\pm\left(w_{0} / w\right)^{m}$. In the 2nd order field the interaction length and the length of the oscillation become shorter than in the paraxial field. When the longitudinal distribution of the field is considered as a wiggler, the 2 nd order field has a shorter period and radiates higher energy photons than does a paraxial field.

as the order of fields increases (Fig. 4); thus this interaction with HOFs increases photon energy.

\section{RESULTS AND DISCUSSION}

The simulation was conducted using previously described code [24] using the laser fields derived in [28,29] up to 7 th order of $\varepsilon$. The dynamics of each electron in an electron bunch was calculated using the relativistic Newton's equation of motion. The radiation was calculated from the dynamics using the relativistic Larmor's formula $[8,9,30]$. The interaction between electrons was ignored because it is much weaker than the interaction between the laser field and electrons.

The simulation produced the temporal structure [Fig. 5(a)] and the spectrum [Fig. 5(b)] of the radiation from the interaction between an electron bunch and a copropagating tightly focused fs laser. The laser had $\mathrm{FWHM}=5 \mathrm{fs}$ and $\lambda=800 \mathrm{~nm}(1.55 \mathrm{eV})$. The laser was focused to a beam waist of $5 \mu \mathrm{m}$ at $z=0$ with an intensity of $2.1 \times 10^{20} \mathrm{~W} / \mathrm{cm}^{2}$ [normalized vector potential $\left.a_{0}=e E_{0} /(m \omega c)=10\right]$. The electron bunch had a radius of $30 \mu \mathrm{m}$, a length of $30 \mu \mathrm{m}$ (or a pulse of $100 \mathrm{fs}$ ), and a normalized emittance of $2 \mathrm{~mm}$ mrad. The energy of the electron bunch was $200 \mathrm{MeV}$ and the energy spread was $0.1 \%$. The electron bunch consisted of $3.0 \times 10^{4}$ electrons which are randomly distributed according to Gaussian distribution throughout the
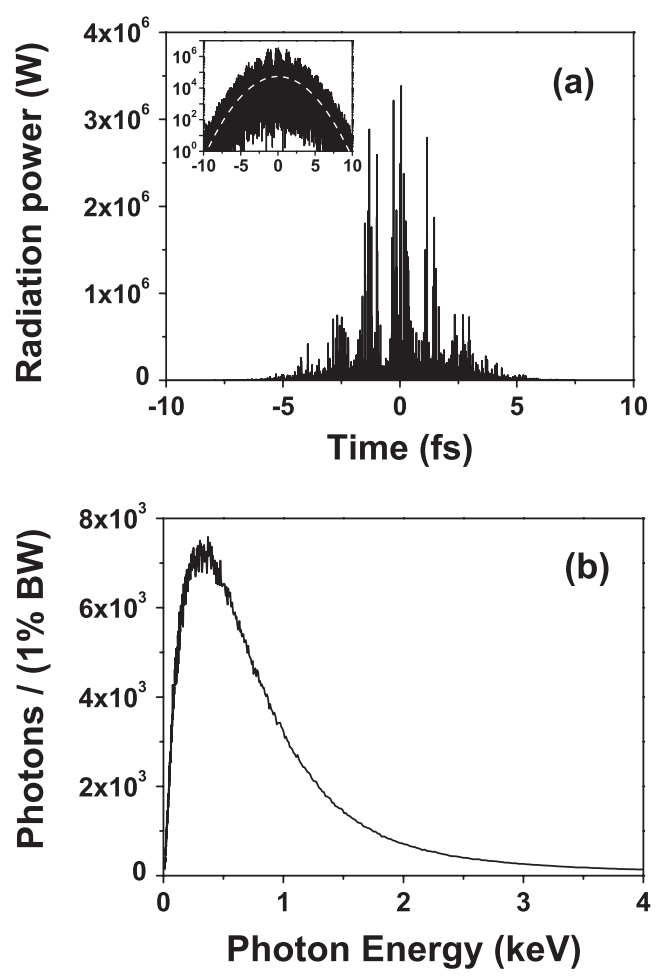

FIG. 5. (a) Temporal structure and (b) spectrum of the radiation from the copropagation interaction between a 5 fs FWHM laser with a beam waist of $5 \mu \mathrm{m}$ and an electron bunch of $200 \mathrm{MeV}$ energy. The laser intensity at focus is $2.1 \times$ $10^{20} \mathrm{~W} / \mathrm{cm}^{2}\left(a_{0}=10\right)$. Inset of (a): the radiation power on a $\log$ scale. White dashed line: best Gaussian fit with a width of $4.83 \mathrm{fs}$ FWHM. The radiation power and the number of photons shown here are those for $0.5 \mathrm{nC}$ electron bunch, which has been scaled up linearly from the results calculated for smaller number of electrons.

bunch. The specifications of the electron bunch used here were chosen from those in Compton backscattering studies [6] and similar quantities were used the freeelectron lasers [22,23,31,32]. The calculations were started at $t=-61 z_{r} / c$ and ended at $t=61 z_{r} / c$, where $t=0$ is defined to be the time at which the centers of the electron bunch meets that of the laser pulse at the center of the focus $(z=0)$. Because the radiations in the current study are not coherent, the radiation power or photon number is linearly proportional to the electron number. The radiation powers and photon numbers were those for a $0.5 \mathrm{nC}$ charge, linearly scaled up from the calculated results. The radiation was detected over the angles $\theta<1 / \gamma$, where $\theta$ is the angle from the $z$ axis (Fig. 2). The duration of the x-ray radiation was 4.83 fs FWHM, which is similar to the width of the laser pulse [inset, Fig. 5(a)].

The duration of the radiation from an electron bunch can be qualitatively estimated as follows. Consider a typical case in which the spatial extent of a laser is smaller than both the confocal parameter and the length of an electron 
beam. A detector measures the radiation from all electrons in the bunch. Each electron in an electron bunch strongly radiates a very short pulse only in the Rayleigh range. The intensity of the pulse from a single electron is almost proportional to the intensity of the laser field at the interaction point in the Rayleigh range. An electron at the peak of the laser intensity in the focal region radiates most strongly. When an electron is away from the strongest point (e.g., by half of the laser pulse width), the radiation intensity would be smaller (a half of the maximum intensity). Therefore, the longitudinal distribution of the radiation strength is similar to the intensity distribution of the laser. The spatial length of the radiation becomes its temporal width. The pulse width of the radiation is then about the same as the laser pulse width or at least of the same order. This analysis indicates that the pulse duration of the radiation is not affected by the length of the electron bunch.

The temporal stability of the radiation source is also important. The laser pulse and the radiated pulse arrive at the detector almost simultaneously. In the tight-focus copropagation scheme, the radiation is generated by a laser in the focal region and the radiated pulse follows the interacting laser pulse at the same speed (the speed of light). This means that the arrival time of the x-ray pulse is independent of the jittering of the electron bunch and that the jitter of the radiation is governed by that of a fs laser pulse $(\sim 100$ as). When an electron bunch does not meet the laser pulse in the Rayleigh range in a copropagating regime, the bunch will not radiate or will radiate very weakly. However, if the pulse length of an electron bunch is larger than its jitter, temporally stable x-ray pulses can be generated for a uniform electron bunch. Typically an electron bunch from a linac has a jitter of $\sim 1 \mathrm{ps}$. The jitter becomes that of undulator-based x-ray source like XFEL. Its jitter can be measured with the resolution of $\sim 60$ fs using the electro-optic sampling [33]. In pump-probe experiments, a tight-focus copropagating scheme provides much finer time resolution than conventional undulator-based sources.

The simulated photon energy spectrum [Fig. 5(b)] suggests that high-energy photons are produced. The total radiated energy [Fig. 6(a)] and the averaged photon energy [Fig. 6(b)] changed as the energy of electron beam changed. To determine the effect of the high-order fields, the simulation was conducted for the 0th field only, for the 0th and 1st fields, for the 0th, 1st, 2nd fields, and for the 0th to 7 th fields. The electron bunch consisted of $7.5 \times 10^{3}$ randomly distributed electrons. The data except photon energy are scaled up for a $0.5 \mathrm{nC}$ bunch from the calculation performed using a smaller number of electrons. The total radiated energy was obtained by integrating of the angular radiation energy over the angles $\theta<1 / \gamma$. The conditions were the same as in Fig. 5, unless otherwise specified.

Figure 6(a) shows the $\gamma$ dependence of the total radiated energies $I_{(0)}, I_{(0-1)}, I_{(0-2)}$, and $I_{(0-7)}$. The fitting to the
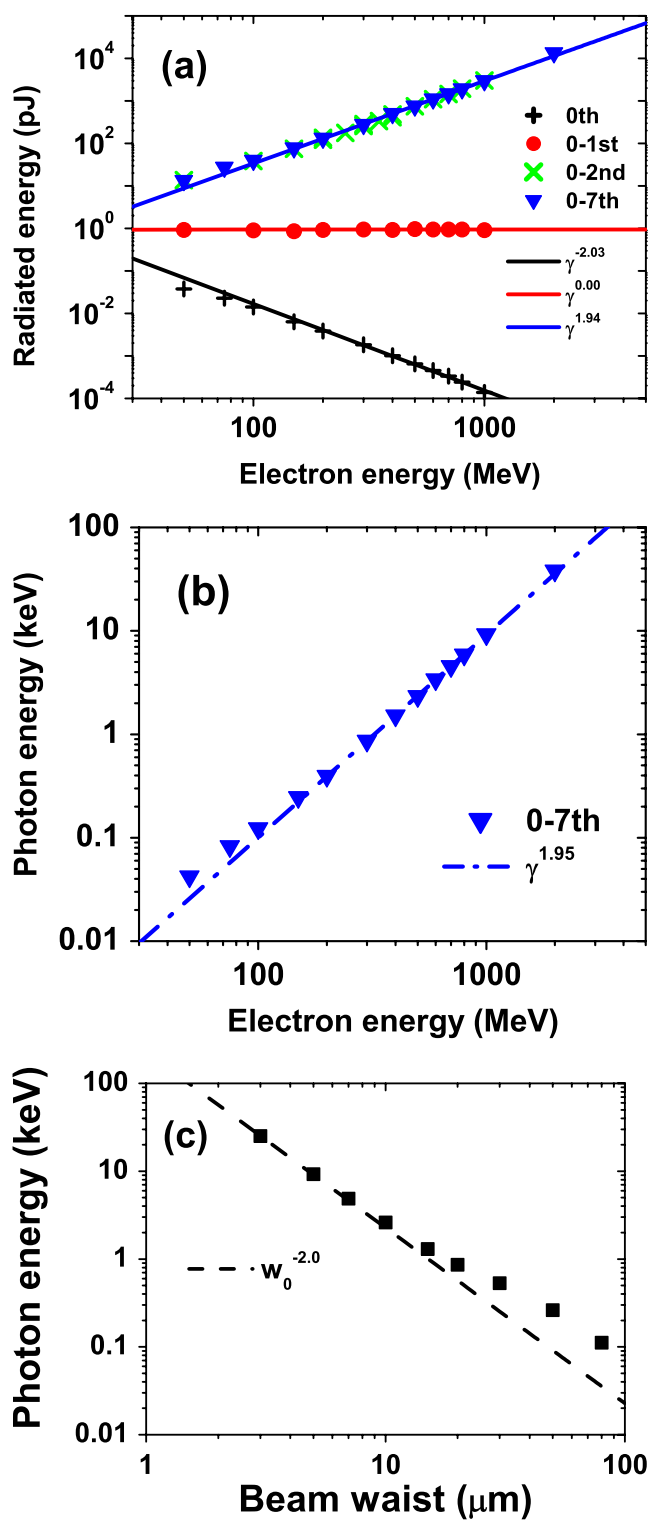

FIG. 6. Dependence of (a) the total radiated energy $I$ with the effect of high-order fields in $\varepsilon$ and (b) the average photon energy $K$ on $\gamma$. (c) Dependence of the average photon energy on $w_{0}$. The normalized vector potential of the laser $a_{0}$ is 10 , beam waist of laser $w_{0}$ is $5 \mu \mathrm{m}$, and the radius of the electron bunch is $30 \mu \mathrm{m}$. The radiation energies are for a $0.5 \mathrm{nC}$ electron bunch, which has been scaled up linearly from results calculated with a small number of electrons. Each point is the average of the four sets of simulations. Standard deviations were always $<5 \%$; error bars are omitted because they are not visible on the log scale.

simulation data shows $I_{(0)} \propto \gamma^{-2.03}$, which is in a good agreement with Eq. (19). In the case of $I_{(0-1)}$ which includes both the 0th and 1st order field, the 1st order field mainly contributes to the radiation so that the total radiated energy $I_{(0-1)}$ becomes independent of $\gamma$ as manifested in Eq. (21). $I_{(0-2)}$, which includes fields up to the 2 nd order, is proportional to $\gamma^{+1.94}$ as shown in Eq. (23), indicating that the 2 nd order field also makes a significant contribution. 
$I_{(0-7)}$ is almost the same as $I_{(0-2)}$, implying that the higherorder fields than the 2 nd order make negligible contributions.

The study of an electron acceleration using a tightly focused laser field by Salamin et al. showed that at least the fields up to the 5th order are required to describe correctly the dynamics of an electron [28]. In that calculation, a low energy $(\gamma<24)$ electron was injected to a focused region at a finite angle $\left(5^{\circ}<\theta<12^{\circ}\right)$ with respect to the laser propagation. Even though the fields higher than the 3rd order are weak, the high-order field can still slightly change the perpendicular position of such a low energy electron. When the focus was tight, the strength of the field is sensitive to the position so the higher-order fields, to a certain degree, change the interaction with the electron somewhat. However, the position of a high-energy electron $(\gamma>100)$, which is effectively heavy, was not changed enough by the higher-order fields.

The average photon energy of the radiation is proportional to square of the electron energy (i.e. $\gamma^{2}$ ) as expected in Eq. (24). The photon energy becomes higher when HOFs were considered. The average radiated photon energy is proportional to the $w_{0}^{-2}$ (or the inverse square of the beam waist) as expected in Eq. (24). Hence, the photon energy can be tuned by changing the beam waist as well as the electron energy. The averaged photon energy is $38 \mathrm{keV}$ when electron energy was $2 \mathrm{GeV}$, but higher photon energy is expected for higher electron energy. Hence, the current scheme can fill the region shorter than $5 \mathrm{fs}$ and higher than $10 \mathrm{keV}$ in Fig. 1.

Because both the photon energy $K$ and the total radiated energy $I$ is proportional to $\gamma^{2}$, the number of radiated photons $I / K$ is independent of $\gamma$. For an electron bunch of $0.5 \mathrm{nC}$ charge, with $a_{0}=10, w_{0}=5 \mu \mathrm{m}$, and a pulse of $5 \mathrm{fs}$ FWHM, the number of radiated photon is $2.1 \times 10^{6}$. For the counterpropagation interaction $\left(180^{\circ}\right.$ collision) between the same electron bunch and laser pulse, the number of the radiated photons is $6 \times 10^{7}$ [6]. Thus, when the HOFs are considered, the number of radiated photon in the copropagation interaction is about $1 / 30$ times that in $180^{\circ}$ collision. Copropagating and counterpropagating geometries produce comparable numbers of photons per unit time, because the radiation pulse width from copropagation interaction $(\simeq 5 \mathrm{fs})$ is $\sim 1 / 20$ as long as that from a counterpropagating collision $(\sim 100 \mathrm{fs})$.

The simulation also shows that the radiation energy increased as the focus spot was reduced. The radiation efficiency (i.e., the radiation energy divided by the energy of the driving laser) varied with the beam waist (Fig. 7). When the laser energy is fixed, tight focusing increases the intensity and normalized vector potential $a_{0}$. When $a_{0}>1$ and is increased, the relativistic effect causes the radiated energy to increase faster than the rate at which intensity is increased [8,34]. To distinguish this relativistic effect from the effect of HOFs, the laser intensity was kept constant for

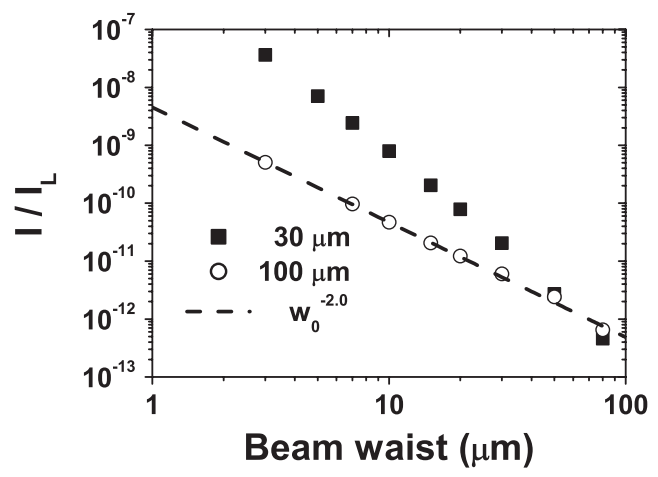

FIG. 7. Change of the radiation efficiency (radiation energy $I$ divided by the energy of the driving laser $I_{L}$ ) with respect to beam waist of $0.5 \mathrm{nC}$ electron bunches of radius $30 \mu \mathrm{m}$ (solid square) and $100 \mu \mathrm{m}$ (open square). The laser intensity was kept constant $\left(a_{0}=10\right)$. The electron bunch of $30 \mu \mathrm{m}$ radius is the same as in Figs. 5 and 6; other specifications of electron bunches are the same as in Figs. 5 and 6.

different beam waists ( $a_{0}=10$ for all data in Fig. 7). If the relativistic effect is ignored, Fig. 7 can be understood as the tendency of the radiating energy itself when the laser energy kept constant. Simulations were performed for electron bunches of radius $30 \mu \mathrm{m}$ and of radius $100 \mu \mathrm{m}$. The radiation efficiency increased as the laser beam waist decreased; therefore reducing focal spot size may increase the flux of photons at a given laser intensity. Also radiation efficiency increased faster than the rate expected in Eq. (23); this is due to the geometrical effect of the Gaussian distribution of electrons in an electron bunch. For a radius of electron bunch of $100 \mu \mathrm{m}$, the radiation efficiency scales as $w_{0}^{-2}$ for $3 \mu \mathrm{m}<w_{0}<20 \mu \mathrm{m}$. In this case, the electron bunch size is sufficiently large compared to the laser beam waist that the electron distribution can be considered as uniform. Then Eq. (23) holds: the total radiation intensity from an electron bunch is independent of the laser beam waist. The laser energy scales as $w_{0}^{2}$; hence, the radiation efficiency scales as $w_{0}^{-2}$. When the size of an electron beam is small (e.g. $30 \mu \mathrm{m})$, the electron distribution cannot be considered uniform: the total radiation intensity is not independent of the laser beam waist but decreases as the laser beam waist increased; hence, the efficiency decreased at a faster rate than $w_{0}^{-2}$ as the beam waist increases.

A series of simulations was also conducted to study how the laser intensity affects the radiation for a fixed beam waist of $5 \mu \mathrm{m}$ in a tight-focus regime. A nonlinear aspect in Thomson scattering such as the generation of high-order harmonics occurs when $a_{0}>1$ [8,34]. The average photon energy increased rapidly when $a_{0}>1$ when electron energy was $50 \mathrm{MeV}$ [Fig. 8(a)]. However, at higher electron energies (i.e., $200 \mathrm{MeV}, 1 \mathrm{GeV}$ ), the average photon energy increased at higher laser energy: for $1 \mathrm{GeV}$, the averaged photon energy began to change when $a_{0}>10$; in the range of $1<a_{0}<10$, the change of the spectrum shape was too 

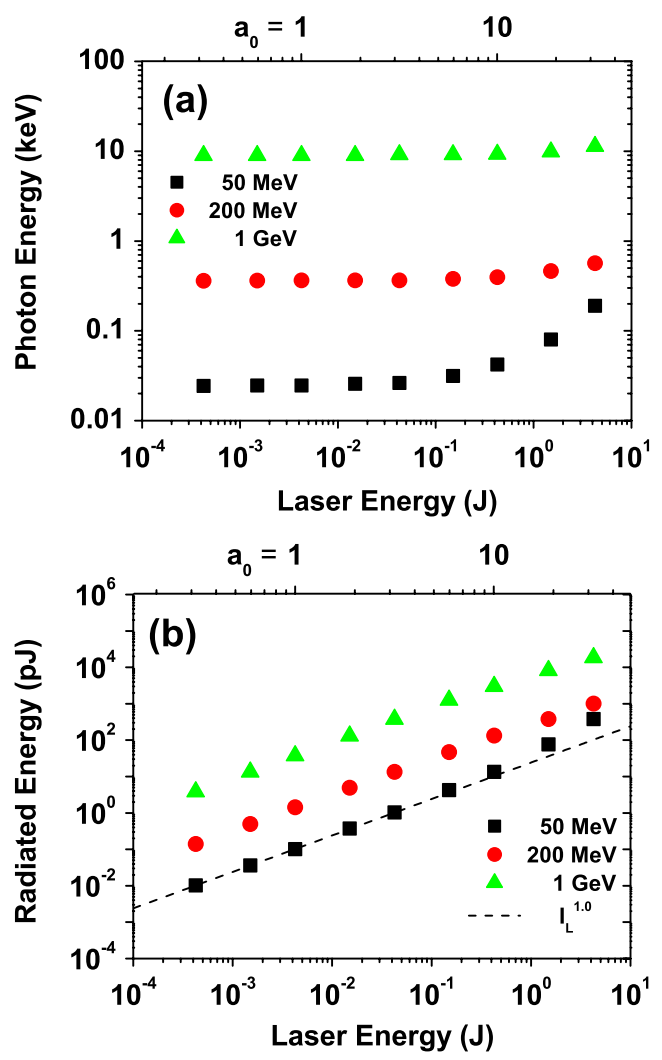

FIG. 8. Dependence of (a) averaged photon energy and (b) total radiation energy on the laser energy for various electron energies. The upper horizontal axis indicates the normalized vector potential, $a_{0}$, which corresponds to the laser energy in the lower horizontal axis. The dashed line of (b) is a fitting line proportional to the first power of laser energy.

weak to change the average. Total radiation energy changed with the laser energy [Fig. 8(b)]. Generally, the radiation energy was proportional to the laser energy except for a few points. When the electron energy was low $(50 \mathrm{MeV})$ and the laser intensity was high $\left(a_{0}>10\right)$, the radiation energy increased due to the relativistic effect.

\section{CONCLUSION}

Simulations suggest that a strong femtosecond x-ray pulse is produced when an electron bunch interacts with a high-intensity tightly focused femtosecond laser pulse in a copropagation geometry. The radiated pulse duration was almost the same as the laser pulse width, which can reach FWHM $\leq 5$ fs; this $\leq 1 / 20$ of the pulse width of the radiation in the counterpropagation interaction can be achieved using current technology. In spite of the time jitter of electron bunches $(\sim 1 \mathrm{ps})$, temporally stable radiation pulses (jitter $\sim 100$ as) can be generated by this scheme.

Simulations suggest that when a laser is focused tightly to the order of its wavelength, the HOFs significantly affect the dynamics of electrons: they increased radiation intensity by several orders of magnitude. The 2 nd order field had an important effect on simulated radiation increase. For a fixed beam waist, the radiation energy and the average photon energy are proportional to $\gamma^{2}$. The calculated number of photons per electron bunch and laser pulse was $2.1 \times 10^{6}$ when an $800 \mathrm{~nm}, 5$ fs FWHM, $420 \mathrm{~mJ}$ laser pulse was focused to a beam waist of $5 \mu \mathrm{m}$ and interacted with a $100 \mathrm{fs}, 0.5 \mathrm{nC}$ electron bunch. The radiation efficiency scaled as the inverse of the 2 nd (or higher) power of the beam waist. The photon energy was proportional to $w_{0}^{-2}$. The result suggests that (i) the radiation efficiency increases as the beam waist decreases, and (ii) the average photon energy of the radiation can be tuned by changing the electron energy or the beam waist. The photon energy can reach $\sim 40 \mathrm{keV}$ for an electron energy of $2 \mathrm{GeV}$ and a laser beam waist of $5 \mu \mathrm{m}$. The physical scheme suggested in this paper can be used to produce an ultrafast $(\sim 5 \mathrm{fs})$ $\mathrm{x}$-ray source in the range of 10 to $100 \mathrm{keV}$ with very small time jitter $(\sim 100$ as $)$.

\section{ACKNOWLEDGMENTS}

This research was supported in part by Global Research Laboratory Program (Grant No. 2009-00439) and by Leading Foreign Research Institute Recruitment Program (Grant No. 2010-00471) through the National Research Foundation of Korea (NRF) funded by the Ministry of Education, Science and Technology (MEST). This research was also in part supported by Basic Science Research Program through the National Research Foundation of Korea (NRF) funded by the Ministry of Education, Science and Technology (Grant No. 20090076001).

[1] N. Bloembergen, Rev. Mod. Phys. 71, S283 (1999).

[2] Available from http://tesla.desy.de/new_pages/TDR_CD/ PartV/fel.html.

[3] K. J. Gaffney and H.N. Chapman, Science 316, 1444 (2007).

[4] K. Sokolowski-Tinten et al., Nature (London) 422, 287 (2003).

[5] R. Neutze, R. Wouts, D. Spoel, E. Weckert, and J. Hajdu, Nature (London) 406, 752 (2000).

[6] F. V. Hartemann et al., Phys. Rev. ST Accel. Beams 8, 100702 (2005).

[7] Y. Ueshima, Y. Kishimoto, A. Sasaki, and T. Tajima, Laser Part. Beams 17, 45 (1999).

[8] K. Lee, Y. H. Cha, M.S. Shin, B. H. Kim, and D. Kim, Phys. Rev. E 67, 026502 (2003).

[9] K. Lee, Y.H. Cha, M. S. Shin, B.H. Kim, and D. Kim, Opt. Express 11, 309 (2003).

[10] K. T. Phuoc et al., Phys. Plasmas 14, 080701 (2007).

[11] A. Rousse et al., Phys. Rev. Lett. 93, 135005 (2004).

[12] R. W. Schoenlein, Science 287, 2237 (2000).

[13] P. Beaud et al., Phys. Rev. Lett. 99, 174801 (2007). 
[14] S. Khan, K. Holldack, T. Kachel, R. Mitzner, and T. Quast, Phys. Rev. Lett. 97, 074801 (2006).

[15] Available from http://xfel.desy.de/.

[16] Available from http://www-ssrl.slac.stanford.edu/lcls/ science.html.

[17] D. Normile, Science 314, 751 (2006).

[18] P. Emma, K. Bane, M. Cornacchia, Z. Huang, H. Schlarb, G. Stupakov, and D. Walz, Phys. Rev. Lett. 92, 074801 (2004).

[19] A. A. Zholents and W. M. Fawley, Phys. Rev. Lett. 92, 224801 (2004).

[20] E. L. Saldin, E. A. Schneidmiller, and M. V. Yurkov, Opt. Commun. 239, 161 (2004).

[21] A. A. Zholents and G. Penn, Phys. Rev. ST Accel. Beams 8, 050704 (2005).

[22] M. Abo-Bakr et al., The BESSY Soft X-ray Free Electron Laser, Technical Design Report, 2004 (BESSY, Berlin, 2004), p. 13.

[23] Claudio Pellegrini and Joachim Stohr, X-ray Free-Electron Lasers: Priciples, Properties and Applications (SLAC, Menlo Park, CA, 2002), p. 4.
[24] H. Lee, S. Chung, K. Lee, and D. Kim, New J. Phys. 10, 093024 (2008).

[25] K. Lee, S. Y. Chung, S. H. Park, Y. U. Jeong, and D. Kim, Europhys. Lett. 89, 64006 (2010).

[26] L. W. Davis, Phys. Rev. A 19, 1177 (1979).

[27] J. P. Barton and D. R. Alexander, J. Appl. Phys. 66, 2800 (1989).

[28] Y. I. Salamin and C. H. Keitel, Phys. Rev. Lett. 88, 095005 (2002).

[29] Y. I. Salamin, Appl. Phys. B 86, 319 (2007).

[30] J.D. Jackson, Classical Electrodynamics (Wiley, New York, 1999), 3rd ed., Chap. 14.

[31] M. Abd-Elmeguid et al., TESLA Technical Design Report: Part V. The X-ray Free Electron Laser (DESY, Helmholtz, Germany, 2001), p. 194.

[32] Available from: http://xfel.desy.de/technical_information/ electron_beam_parameter/.

[33] A. L. Cavalieri et al., Phys. Rev. Lett. 94, 114801 (2005).

[34] E. Esarey, S. K. Ride, and P. Sprangle, Phys. Rev. E 48, 3003 (1993). 